\title{
Glucose tolerance female-specific QTL mapped in Collaborative Cross mice
}

Hanifa J. Abu-Toamih Atamni ${ }^{1}$, Yaron Ziner ${ }^{2}$, Richard Mott ${ }^{3}$, Lior Wolf ${ }^{2}$ and Fuad A. Iraqi ${ }^{1}$

'Department of Clinical Microbiology and Immunology, Sackler Faculty of Medicine, Tel-Aviv University, Israel. ${ }^{2}$ The Blavatnik School of Computer Science; Tel-Aviv University, Israel.

${ }^{3}$ Department of Genetics, University Collage of London, London, UK.

Hanifa J. Abu-Toamih Atamni: hanifajalal@hotmail.com

Yaron Ziner: ziner.yaron@gmail.com

Richard Mott:

r.mott@ucl.ac.uk

Lior Wolf: liorwolf@gmail.com

Fuad A. Iraqi:

fuadi@post.tau.ac.il

\section{Corresponding author:}

Prof. Fuad A. Iraqi

Chairman, Department of Clinical Microbiology and Immunology

Sackler Faculty of Medicine

Tel Aviv University, Ramat Aviv

Tel Aviv 69978

Israel

Email: fuadi@post.tau.ac.il

Running title: collaborative cross mice, high-fat diet, type 2 diabetes, Area under curve, QTL mapping, females-dependent QTL. 
Introduction: Type 2 diabetes (T2D) is a complex metabolic disease characterized by impaired glucose tolerance. Despite environmental high risk factors, host genetic background is a strong component of T2D development. Herein, novel highly genetically diverse strains of Collaborative Cross (CC) mice were assessed to map quantitative trait loci (QTL) associated with variations of glucose tolerance response.

Materials \& Methods: Total 501 mice of 58 CC lines were maintained on high-fat (42\% fat) diet for 12 weeks. Thereafter, an Intraperitoneal Glucose Tolerance Test (IPGTT) was performed for 180 minutes. Subsequently Area under curve for the glucose values at zero and 180 minutes (AUC0-180), were measured, and used for QTL mapping. Heritability and coefficient of variations in glucose tolerance $(\mathrm{CVg})$ were calculated.

Results: One-way analysis of variation (ANOVA) was significant $(\mathrm{P}<0.001)$ for AUC0-180 between the CC lines as well between both sexes. Despite Significant variations for both sexes, QTL analysis was significant, only for females, reporting a significant females-dependent QTL ( 2.5 Mbp) associated with IPGTT AUC0-180 trait, located on Chromosome 8 (32-34.5 Mbp, containing 51 genes). Gene browse revealed QTL for body weight /size, genes involved in immune system and two main protein-coding genes involved in the Glucose homeostasis, Mboat4 and Leprotl1. Heritability and coefficient of genetic variance $(\mathrm{CVg})$ were 0.49 and 0.31 for Females, while for males 0.34 and 0.22 , respectively.

Conclusions: Our findings demonstrates the role of genetic factors controlling glucose tolerance that significantly differ between sexes requiring independent studies for females and males towards T2D prevention and therapy. 


\section{Introduction}

Type 2 diabetes (T2D) is one of four leading causes of no communicable diseases (NCD) deaths in 2012, while according to the World Health Organization (WHO) report for 2014; 1.5 million deaths of a total 38 million in 2012 were caused by diabetes. The annual number of deaths due to noncommunicable diseases is steadily increasing (unlike infectious diseases) and projected to increase from 38 million in 2012 to 52 million by 2030 , whereas Diabetes is predicted to be $7^{\text {th }}$ cause of death (increase 2-3 places in rank from 2012 to 2030) (Mathers and Loncar 2002). Etiology of T2D is widely diverse involving multiple environmental (lifestyle, physical activity, stress, diet, age etc.) and genetic factors and their complex interactions. Type 2 diabetes is one of the most ancient known human diseases (Ahmed 2002), characterized by complex pathogenesis and despite improvement through the years in T2D prevention and therapies via lifestyle and pharmacological intervention (Stevens 2015), the genetic architecture of the disease is still unexplained. Susceptibility towards development and progress of Type 2 diabetes differ between ethnic and ancestry (genomic heritage) groups in response to common environmental conditions. Individuals from different racial or ethnic groups experience the disease differently at the level of co-morbidities, and at the level of Glycemic control despite socioeconomic status or health insurance availability (Saydah et al. 2007; Harris et al. 1999; Qi at al. 2008). Since the introduction of genomewide association studies (GWAS), considerable efforts have been invested in exploring genes-environment interactions towards higher risk of T2D. Diet-gene interactions were one of the leading studies among populations, several studies showed that Westernized dietary patterns had been associated with higher risk of impaired glucose tolerance and T2D (Van Dam et al. 2002; Gittelsohn et al. 1998; Qi and Liang 2010). To date, GWAS studies revealed approximately 88 genetic loci associated with $\mathrm{T} 2 \mathrm{D}$ and 83 related with glycemic traits (glucose, $\mathrm{HbA1C}$, insulin, and proinsulin) (Mohlke and Boehnke 2015). Dissecting the genetic architecture of T2D development and progress is crucial for the invention of personalized genetic risk prediction tools and discovery of new 
therapeutic implementations among populations (Bao et al. 2013). Major limitation of the human GWAS is lack of proper, controlled, environmental factors, which might generate biased results. Therefore, using the mouse model in the basal studies of complex diseases is widely accepted, where researchers can control the environmental factors while scanning mice from different genetic backgrounds. In this context, T2D is considered a complex trait disease, where the environmental challenge is the Western diet. The mouse model resource used in this study is the new Collaborative Cross (CC), a unique model for dissecting QTL of host susceptibility towards the development of T2D in response to high-fat ( $42 \%$ Fat) dietary challenge. The CC population was created by a community effort of the complex trait consortium (CTC, www.complextrait.org) (Churchill et al. 2004; Iraqi et al. 2012; Threadgill and Churchill 2012). This unique genetic resource comprises a set of approximately 300 recombinant inbred lines (RIL) that were created by full reciprocal 8-way matings of eight divergent strains of mice: A/J, C57BL/6J, 129S1/SvImJ, NOD/LtJ, NZO/HiLtJ, CAST/Ei, PWK/PhJ, and WSB/EiJ. Controlled randomization and minimization of selection during the breeding process will recombine the natural genetic variation present in these inbred strains. The result is a unique collection of RIL exhibiting a large phenotypic and genetic diversity, and brings the tremendous genetic variation potential of the mouse inbred lines to phenotypic expression (Paterson 1995; Churchill et al. 2004). Full details of CC lines and their power of dissecting and mapping QTL associated with host susceptibility to complex traits, are presented (Iraqi et al. 2008; Durrant et al. 2011; Aylor et al. 2011; Philip et al. 2011; Iraqi et al. 2012; Shusterman et al. 2013; Iraqi et al. 2014; Soller and Iraqi 2014; Vered et al. 2014; Lore et al. 2015; Levy et al., 2015; Abu Toamih-Atamni et al. 2016a\&b; Dorman et al. 2016).

Herein we demonstrate exclusive use of the $\mathrm{CC}$ unique mouse reference population to identify QTL and subsequently genes associated with host glucose tolerance in response to high fat diet. 


\section{Materials and Methods}

\section{Ethical statement}

Mice were housed in the small animal facility of the Sackler faculty of medicine at Tel-Aviv University (TAU) according to standard protocol approved by the animal use and care committee at TAU (approved experiment number $\mathrm{M}-12$ 025).

\section{Housing and Diet}

Mice were housed, separately by sex and CC line on hardwood chip bedding in open-top cages, 12 hours light/dark cycle (6:00 am $-6: 00 \mathrm{pm})$ at $21-23^{\circ} \mathrm{C}$, given tap water and standard rodent chow diet ad libitum (TD.2018SC, Teklad Global, Harlan Inc., Madison, WI, USA; containing \%Kcal from Fat 18\%, Protein $24 \%$, Carbohydrates $58 \%$ ), since weaning at age of 3 weeks old, until start of the experiment at age of 8 weeks old. To induce the development of diabetogenic response, mice were fed by a purified experimental high-fat diet (TD 88137 Harlan Teklad, Madison, WI, USA; containing $42 \%$ of calories from fat and $34.1 \%$ from carbohydrate, primarily sucrose) from age of 8 to 20 weeks old.

\section{CC lines}

All the CC lines used in this study are being developed at TAU small animal facility. CC lines are developed by full-sib mating technique to exceed currently inbreeding generation 16 (G16), further details of the TAU CC colony availablein previous reports (Iraqi et al. 2008; Iraqi et al. 2012). In the current study, we used 501 mice generated from 58 CC lines, with representation of both sexes. In details, our study cohort consisted of 200 female mice from 44 CC lines and 301 male mice from 56 CC lines. Sex presentation was not equal for all CC lines due to breeding variations between lines. 


\section{Genotype}

All CC lines were genotyped with high density SNP markers, as previously described, and used (Yang et al. 2009; Iraqi et al. 2012; Vered et al. 2014; Levy et al. 2015; Abu Toamih-Atamni et al. 2016b). Briefly, all SNPs were filtered out with heterozygous or missing genotypes in the 8 CC founders, or that were not in common between the arrays. The SNPs were mapped onto build 37 of the mouse genome. The HAPPY HMM computed a descent probability distribution for each of the used SNPs.

\section{Phenotype}

Diabetogenic effect in response to high-fat dietary challenge was measured by the intraperitoneally glucose tolerance test (IPGTT) (Montgomery et al. 2013; Leiter et al. 2009) after 12 weeks dietary challenge. For the IPGTT, mice were fasted for 6 hours (6:00am-12:00am); with free access to water. Subsequently, fasting blood glucose levels were determined at time 0 , and then a solution of glucose (2.5gr glucose per $\mathrm{Kg}$ mouse) was administered by intra-peritoneal (IP) injection and blood glucose levels were monitored for 180 minutes (time 0, 15, 30, 60, 120 and 180 minutes). Blood glucose levels were measured from tail vein using U-RIGHT glucometer TD-4267 (TaiDoc Technology Corporation 3F, 5F, No.127, Wungong $2^{\text {nd }}$ Rd., 24888 Wugu Township, Taipei County, Taiwan). Quantitative glucose clearance ratio of the total 180 minutes IPGTT was calculated using area under curve (AUC) trapezoid model from time zero (0) to time 180 minutes, as below:

${ }^{A U C}{ }_{0-180}=180$ minutes * (Glucose level at time $0+$ Glucose level at time 180)/2

Full details of the recorded traits on these mice are presented in Abu ToamihAtamni et al. 2016a.

\section{Statistical Analysis}


Data analysis was performed using a statistical software package IBM SPSS statistics 23. One-Way ANOVA was carried out for testing the significance of the AUC0-180 means variations between the different CC lines, a $P$ value of 0.05 or less was considered significant.

\section{Heritability and coefficient of genetic variation}

Heritability $\left(\mathrm{H}^{2}\right)$ is a statistic that estimates the fraction of phenotypic variance that can be attributed to genetic variance among the population. Here, we used the ANOVA results of the AUCO-180 trait to calculate the broad sense heritability (including epistatic, but not dominance effects) using the $H^{2}$ formula below:

$H^{2}=V_{g} /\left(V_{g}+V_{e}\right)$

Where, $H^{2}$ is the heritability, $V_{g}$ is the genetic variance among $\mathrm{CC}$ lines $\left(\left(\mathrm{MS}_{\text {between }}\right.\right.$ $\left.\left.-V_{e}\right) / n\right)$ and $V_{e}$ is the environment variance $\left(\mathrm{MS}_{\text {within }}=\right.$ variance within CC line).

Following heritability statistic, we calculated the genetic coefficient of variation $\left(\mathrm{CV}_{\mathrm{G}}\right)$, which indicate the absolute amount of genetic variation (between $\mathrm{CC}$ lines). $\mathrm{CV}_{\mathrm{G}}$ was calculated using the result of standard deviation among $\mathrm{CC}$ lines $\left(\mathrm{SD}_{\mathrm{G}}\right)$ and trait mean (AUC0-180) overall CC lines, as below:

$\mathrm{CV}_{\mathrm{G}}=\mathrm{SD}_{\mathrm{G}} /$ Mean

For further details on calculations, see Iraqi et al. 2014.

\section{QTL mapping}

The HAPPY package also tests for the existence of a QTL at each locus using the estimated probabilities of descent from the founder strains to estimate the phenotypic effect attributable to each founder strain. If these effects are significantly different then there is evidence for a QTL. Thus, for the initial stage 
of linkage analysis based on single trait analysis, a standard polyallelic "marker" model will be used. This model tests whether there is an overall association between the marker and the trait by comparing after fitting the complete model including the mean, any fixed effects such as sex and generation, and the marker alleles

\section{Merge analysis}

Testing sequence variations segregation between the $\mathrm{CC}$ founders, using merge analysis to fine-tune the AUC0-180 QTL and identify candidate genes. Analysis methodology will be merged (Yalcin et al. 2005) to test which variants under a QTL peak were compatible with the pattern of action at the QTL. We used the Perlegen SNP database to test sequence variants globally and the Sanger SNP database for individual genes. A successful use of this approach was recently published (Durrant et al. 2011; Vered et al. 2014; Levy et al. 2015; AbuToamih Atamni et al. 2016b).

\section{Genome Browser}

Using the Mouse Genome Informatics (MGI) database resource (http://www.informatics.jax.org/), enabled us to examine the reported QTL in details for genetic features located within the QTL genomic interval, related to glucose homeostasis/metabolism or involved systems in type 2 diabetes, such as immune/endocrine systems. The MGI search revealed known-genes, mRNAs, QTLs and more. Each result suggested at the MGI was further investigated in the literature for biological roles.

\section{Results}


Following 12 weeks of High-Fat dietary (HFD) challenge, the CC lines diabetogenic response was assessed by an Intraperitoneal Glucose Tolerance Test (IPGTT) for 180 minutes. Thereafter, the overall area under curve (AUCO180 ) of the glucose clearance was calculated and used as an indicator for diabetic status, where higher levels of AUC0-180 were associated with slower glucose clearance and even impaired glucose tolerance. Genotypic data of the CC lines was scanned using the HAPPY software for genetic linkage with the trait AUC0-180, first for overall population and then for females and males separately. All results reported in details below, starting from phenotypic data, heritability and $\mathrm{CV}_{\mathrm{g}}$ calculations, to QTL analysis and founders' effect analysis, ending with Identification of candidate genes.

\section{Glucose tolerance significant variations between CC lines}

As shown in Fig.1 (A, B and C), One-way analysis of variation (ANOVA) revealed highly significant variations for AUCO-180 means between CC lines overall population (Fig.1A) $(F(57,443)=4.93, p<0.001)$, also for females (Fig.1B) $(F$ $(43,156)=4.38, p<0.001)$ and males (Fig.1C) $(F(55,245)=3.23, p<0.001)$, separately. The overall CC lines population ( $n=501 / 58$ CC lines) showed mean AUC0-180 of $45515.6( \pm 846.8) \mathrm{mg} / \mathrm{dL}^{*} \mathrm{~min}$, while mean values ranged between IL-2146 representing the lowest value of $24345( \pm 6345) \mathrm{mg} / \mathrm{dL}^{*} \min$ and IL-2452 representing the highest value of 82575 , and IL-2457 located at median point of $45495( \pm 8685) \mathrm{mg} / \mathrm{dL}^{*} \min$.

\section{Females AUC0-180 significantly lower than Males}

From the total assessed $58 \mathrm{CC}$ lines, 42 lines had representation of both sexes (Females $-\mathrm{n}=194$ / Males $-\mathrm{n}=245$ ). Two-way ANOVA was used to assess the significance of sex and line interaction (combined effects) affecting AUC0-180 means variations. ANOVA output revealed a highly significant independent sex 
and line effects $(p<0.001)$, and significant Sex * Line interactions $(p<0.05)$ influencing AUC0-180 variation of means. The reported AUCO-180 means were $35847.1( \pm 1285.6) \mathrm{mg} / \mathrm{dL}^{*} \min$ for females versus $48690.1( \pm 1209.1)$ $\mathrm{mg} / \mathrm{dL}^{*}$ min for males, $\sim 1.4$ folds difference in favor of females at 83\% (35 of 42 CC lines) of the cases and favor of males only at 16\% (7 of 42 CC lines) of the cases (lower $\mathrm{AUC}_{0-180}$ indicates effective glucose clearance). The difference of AUC0-180 means between sexes within each individual CC line, varied in a wide range among the $\mathrm{CC}$ population as shown in Fig. 2 .

\section{Heritability and Genetic coefficient variation}

Heritability estimates have a value between 0 to 1 . As shown in table 1, heritability $\left(\mathrm{H}^{2}\right)$ and genetic coefficient variation $\left(\mathrm{CV}_{\mathrm{G}}\right)$ calculations revealed values of 0.34 and 0.25 , respectively, for the overall CC lines population. When data was split by sex, $\mathrm{H}^{2}$ and $\mathrm{CV}_{\mathrm{G}}$ values were, respectively, 0.49 and 0.31 for females, while 0.34 and 0.22 for males. $\mathrm{H}^{2}$ value of 0.5 and above can be considered as high heritability of the trait hence the reported AUCO-180 $\mathrm{H}^{2}$ value of the females (0.49) is high, indicating that $49 \%$ of the observed variation of AUC0-180 between the CC lines is due to genetic factors. Consequently, $\mathrm{CV}_{\mathrm{G}}$ value of the females reveals that $31 \%$ of the genetic variations among $\mathrm{CC}$ lines were generated by these genetic factors.

Females-dependent QTL associated with AUCO-180 trait and founder effect

Initially, we performed QTL mapping for AUC0-180 trait for the entire population of the 501 mice of the 58 CC lines, including males and females. However, no significant QTL were detected. Subsequently, we performed QTL mapping for male and females, separately. Interestingly, data analysis for male only, did not show any significant QTL. However, the female data, indeed 
showed significant QTL located on Chr8 with genomic interval of $2.5 \mathrm{Mbp}$ (32.02-34.52 Mbp) $(\log P=5.9)$ as presented in Fig.3. The threshold based on permutation test is presented in figure and found to be $-\log P=5.65$.

\section{Founder effect}

We evaluated the effect of each founder genotype at the females AUC0-180 mapped QTL interval, and estimated to the assessed trait. Results of this analysis are presented for the QTL in Fig.4. The locus showed complex pattern of haplotype effects of the founders, with the wild-derived strains (mainly PWK) playing a major role on increase AUC values, while NZO genotype reduce this trait, although other strains also contributed (positive or negative) to the overall QTL effect.

\section{Merge analysis}

Testing sequence variations segregation between the CC founders, using merge analysis to fine-tune the AUC0-180 QTL and identify candidate genes. Fig.5 shows the merge analysis, and interestingly our results have shown that the majority of the SNPs within the haplotype QTL interval are highly significant and enabled higher resolution of the QTL peak location ( $\log P$ score $=5.9$, threshold 5.65 at $P$-value $<0.05$ ).

\section{Identification of candidate genes within the mapped QTL interval}

The reported QTL contains 13 protein-coding genes, 10 QTLs and other genetic features (non-coding RNA/ heritable phenotypic markers/gene segments/ pseudogenes). The protein coding genes with most relevance to glucose 
tolerance were related to immune system, endocrine system and regulation of glucose homeostasis.

With most relevance to the glucose homeostasis traits, we may suggest genes involved in regulation glucose homeostasis, such as Mboat4 (MGl:2685017), named membrane bound 0 -acyltransferase domain containing 4, which encodes enzymes that transfer organic acids, typically fatty acids, onto hydroxyl groups of membrane-embedded targets (Hofmann 2000). MBOAT4 mediates the octanoylation of the stomach hormone ghrelin (GOAT - Ghrelin $O$ Acyltransferase), which plays endocrine role in the regulation of glucose homeostasis (Yang et al. 2008; Kirchner et al. 2013).

Another major candidate gene is Leprotl1 (MGl:1915442), named leptin receptor overlapping transcript-like 1 , plays a role in the control of hepatic Growth Hormone $(\mathrm{GH})$ resistance to involved between nutritional signals and $\mathrm{GH}$ actions on body growth and metabolism. LEPROTL1 expression is regulated in the mouse liver by physiologic and pathologic changes in glucose homeostasis (Touvier et al. 2009). As well, we observed the Wrn gene (MGI:109635), named Werner syndrome homolog (human) and involved in age-related osteoporosis, adipose tissues/ cardiovascular system/ digestive/ growth/ size/ body/ immune system/ mortality/ aging (Tracy et al. 2014).

Furthermore, the MGI search revealed candidate genes involved in the immune system, and might be indirectly related to the Type 2 diabetes progress as an inflammatory disease. One of the observed genes was the Gsr gene, named glutathione reductase (MGI:95804), and had been shown to be involved in immune response against bacterial challenge (phagocytes), catalyzes the reduction of glutathione disulfide to glutathione (Yan et al. 2012), alongside additional functionaries in Cardiovascular System / vision eye (Blackshaw et al. 2004), also crucial for limiting tissue damage (Rogers et al. 2006) which are highly engaged to T2D metabolic syndrome health complication. 
Saraf gene (MGI:1915137), named store-operated calcium entry-associated regulatory factor, involved inter alia in the Endocrine system, rather in the Pancreas development (Hoffman et al. 2008), the major organ of the body that respond to glucose levels and plays main role in glucose homeostasis.

In addition to protein coding genes, ten QTL feature types were exposed, located within our QTL genomic interval; eight of the ten QTLs were with relevance to our phenotype and therefore mentioned here. Three named Egq12 (early growth QTL 12), W10q14 (weight 10 weeks QTL 14) and W6q9 (weight 6 weeks QTL 9) were related to Growth and body size (Rocha et al. 2004). Another three QTLs named Eae36 (experimental allergic encephalomyelitis susceptibility 36) (MazónPeláez et al. 2005), Hbnr12 (Heligmosomoides bakeri nematode resistance 12) (Behnke et al. 2010) and Pgia4 (proteoglycan induced arthritis 4) (Otto et al. 1999), related to different immune system responses. Following the previously mentioned order of the immune system QTLs, the first related Inflammatory demyelinating disease of the nervous system (EAE), the second related to Immune response to infection (Heligmosomoides bakeri), and the third associated with autoantibody production in the context of rheumatoid arthritis (RA) study in the murine model Proteoglycan-induced arthritis (PGIA). Two additional QTLs were related to known T2D high risk co-morbidities, one associated with bone mineral density, Bmd39 (sex-specific), named bone mineral density 39, and linked to bone geometry and strength (Robling et al. 2007). Previous studies reported osteopenia and osteoporosis complications of T2D diabetes (Mathen et al. 2015), even though several studies shows that BMD levels were not affected by T2D (Caglayan et al. 2015), the incidence of bone fracture is increased among T2D patients, suggesting that quality of the bones might still be affected by the disease (Carnevale 2014). The second comorbidity was associated with tumor genesis of colon cancer, Scc8, named colon tumor susceptibility 8 (van Wezel et al. 1999). According to studies and most recent meta-analysis diabetic patients have an increased risk of colon cancer compared to non-diabetic (Guraya 2015). 
Finally, Human - Mouse: Disease Connection (HMDC) search for the given QTL genomic location revealed about 18 gene homologs from 28 phenotypes/diseases. Out of this list, directly relevant to glucose tolerance trait, six genes (GSR/Leprotl1/Mboat4/Nrg1/Tex15 and WRN) _ with homologs in human are suggested playing important role in the homeostasis/metabolism system.

\section{Discussion}

Herein, we demonstrate, for the first time, the implementation of the unique Collaborative Cross (CC) mouse model to study the complex genetic architecture of type 2 diabetes in response environmental dietary challenge of high-fat diet (42\%). The accumulated data of T2D studies proves repeatedly the complexity of the disease etiology, once attributed mainly to environmental risk factors, and today is known to be controlled by numerous geneticsenvironment interactions. Therefore, massive efforts are invested for dissecting the genetic architecture of T2D, which will restrain the alarming predictions for T2D epidemic, first at the level of quick diagnosis using genetic biomarker and second at the level of personalized medicine. Achieving this challenge requires great efforts, collaboration of scientists and clinicians and the realization that T2D differ among populations and should be defined into categories based on genetic and environmental factors.

Human GWAS survey revealed, so far, at least 88 genetic loci associated with T2D and 83 related with glycemic traits (Mohlke and Boehnke 2015), require further study to understand their biological role and mechanism involved in T2D. Interestingly, none of the genes, which were mapped within our QTL interval, identified in the human mapped genes. Therefore, we considered our results to be novel by identifying new genes associated with the tested trait. Human GWAS methodologies ameliorate in recent years with evident success to unravel phenotype-genotype associations, nevertheless, this powerful tool arises several 
limitations mainly due to lack of proper control and constraints of further trials in human population under controlled environments for investigation of candidate genes biological roles/functioning. Hence, using an animal model for human diseases study was dedicated to overcome these limitations. Particularly for GWAS studies, the mouse model is widely used with notable potential due to many advantages, including availability of mouse genomic data resources, availability of genetic experimental tools, ease of breeding and ability of strictly controlled environmental conditions (Cox and Church 2011). For this purpose, the Collaborative Cross (CC) mouse population was designated, representing a model for heterogeneous human population for dissecting phenotypic-genotypic association underling differences among population towards common environmental exposure. In the present study, the CC lines corresponds for heterogeneous population and high-fat diet (HFD - 42\% fat) corresponds for T2D risk environmental challenge, whereas QTL mapping analyzes the linkage between diabetogenic phenotype (impaired glucose tolerance measured as AUC0-180) and genotypic components. Once a QTL mapped, the next level will be search for candidate genes, validation of candidate gene and functioning pathways, towards translation to advanced level of human genetics, a numerous efforts invested by extensive collaborations of basic and clinical researchers.

In the current study, QTL mapping revealed a new sex-specific loci standing for total Area Under Curve in response to HFD, calculated for the area under curve between start time point to end time point of the 180 minutes Intraperitoneal Glucose Tolerance Test (IPGTT). Eventually, data processing exposed various patterns of glucose clearance process that differ significantly between the CC lines in response to the same environmental conditions. Additionally, a significant sex effect was observed for the overall CC lines population, in which AUC0-180 of Males was higher than Females, indicating greater glucose clearance impairment in response to HFD for males, i.e. higher sensitivity to HFD induced glucose-impaired tolerance than in females. Sex difference was significant as an overall trend but varied between the $\mathrm{CC}$ lines, when examined within each CC line. 
The reported QTL, is a females-dependent, named AUC0-180 QTL ( 2.5 Mb) and mapped at genomic location of Chr8:32.02-34.52 Mb. Merge analysis enabled higher resolution of the QTL peak location $(\log P=5.9$, threshold Pvalue $<0.05$ ), thereafter we scanned the observed interval for candidate genes using the international database resource for the laboratory mouse, Mouse Genome Informatics (MGl; http://www.informatics.jax.org). The scanned interval included 10 QTL and 50 genes, of which 13 genes were protein-coding genes, studied with known functions. Amongst the 13 protein coding genes, Mboat4 (MGI: 2685017) and Leprotl1 (MGI: 1915442) reported to be highly involved in regulation of glucose homeostasis, an evidence to support the significance of the AUC0-180 association with glucose tolerance phenotype. As well, 8 out of the mentioned 10 QTL were related to phenotypes of high relevance to impaired glucose tolerance and T2D co-morbidities, such as body weight and size, immune response, bone mineral density and colon cancer. Recently, we have published results of mapping QTL for females, only with non-alcoholic fat liver accumulations (Abu Toamih-Atamni et al. 2016b). It is believed that there are different mechanisms that control obesity and related diseases including NAFLD and blood glucose in males and females.

Furthermore, we searched the AUC0-180 interval for Human-Mouse Disease Connection (HMDC; http://www.informatics.jax.org/humanDisease.shtml) to identify possible connections of our findings in the mouse genome to corresponding genetic findings reported in the human disease. Search results revealed 5 gene homologs in humans (Wrn/ Tex15/Ppp2cb/Gsr/Mboat), where the genes Wrn and Mboat associated with phenotypes of growth/size/body and homeostasis/metabolism pathways. Two of the 5 mentioned homologous genes, Wrn and Gsr, were associated with known human diseases, Werner Syndrome and Glutathione Reductase disease, respectively. Werner Syndrome, attributed to Wrn protein coding gene, is characterized by wide range of metabolic/growth complications, including development of T2D. Glutathione Reductase disease, attributed to Gsr protein coding gene deficiency leading to multiple complications including Cardiovascular system and cataract development, which are known as main complication also in T2D. Altogether, genes search of AUCO- 
180 interval corroborates the significance of our findings to be relevant to T2D direct/indirect pathways, along with the suitability of using AUCO-180 as diabetogenic phenotype.

Finally, in previous studies researchers, usually focus on impaired glucose clearance, but less on QTL associated with improved glucose clearance. This report is one of the few studies that addresses both, impaired glucose clearance phenomenon and QTL associated with this trait.

\section{Acknowledgements}

The present work is part of a Ph.D. thesis by Hanifa J. Abu-Toamih Atamni. The authors declare no competing financial interests or other associations that might pose a conflict of interest (e.g., pharmaceutical stock ownership, consultancy). This work was supported by the Hendrech and Eiran Gotwert Fund for studying diabetes, Wellcome Trust grants 085906/Z/08/Z, 075491/Z/04, Wellcome Trust core funding grant 090532/Z/09/Z, and core funding by TelAviv University. 


\section{References:}

Ahmed AM (2002) History of diabetes mellitus. Saudi Med J 23:373-378.

Aylor DL, Valdar W, Foulds-Mathes W, Buus RJ, Verdugo RA et al (2011) Genetic analysis of complex traits in the emerging Collaborative Cross. Genome Res 21: 1213-1222.

Abu Toamih-Atamni HJ, Mott R, Soller M and Iraqi FA (2016a) High-fat induced development of increased fasting glucose levels and impaired response to intraperitoneal glucose challenge in collaborative cross mouse reference population. BMC Genetics 17:10.

Abu Toamih-Atamni H, Botzman M, Mott R, Gat-Vicks I and Iraqi FA (2016b) Mapping Quantitative Trait Loci associated with host susceptibility to nonalcoholic fat liver accumulations using collaborative cross mouse genetic reference population. Mammalian Genome Journal. July 15: [Epub ahead of print]: DOI: $10.1007 / \mathrm{s} 00335-016-9658-3$.

Bao W, Hu FB, Rong S, Rong Y, Bowers K et al (2013) Predicting risk of type 2 diabetes mellitus with genetic risk models on the basisof established genomewide association markers: a systematic review. Am JEpidemiol 178:1197-1207.

Behnke JM, Menge DM, Nagda S, Noyes H, Iraqi FA et al (2010) Quantitative trait loci for resistance to Heligmosomoidesbakeri and associated immunological and pathological traits in

mice: comparison of loci on chromosomes 5, 8 and 11 in F2 and F6/7 intercross lines of mice. Parasitology 137:311-320.

Blackshaw S, Harpavat S, Trimarchi J, Cai L, Huang H et al (2004) Genomicanalysis of mouse retinal development. PLoS Biol 2:E247. 
Caglayan EK, Engin-Ustun Y, Sari N, Karacavus S, Seckin L et al (2015) Evaluation of bone density measurement in type 2 diabetic postmenopausal women with hypertension and hyperlipidemia. J Menopausal Med 21:36-40.

Carnevale V, Romagnoli E, D'Erasmo L, D'Erasmo E (2014) Bone damage in type 2 diabetes mellitus. NutrMetabCardiovasc Dis 24:1151-1157.

Churchill G, Airey D, Allayee H, Angel J, Attie A. et al (2004) Complex Trait Consortium. The Collaborative Cross, a community resource for the genetic analysis of complex traits. Nat Genet 36: 1133-1137.

Cox RD, Church CD (2011) Mouse models and the interpretation of human GWAS in type 2 diabetes and obesity. Dis Model Mech 4:155-164.

Dorman A, Daria Baer, Tomlinson I, Mott R and Iraqi FA (2016) Genetic Analysis of Intestinal polyp development in Collaborative Cross mice carrying the ApcMin/+ mutation. BMC Genetics 17:46.

Durrant C, Tayem H, Yalcin B, Cleak J, Goodstadt L et al (2011) Collaborative Cross mice and their power to map host susceptibility to Aspergillus fumigatus infection. Genome Res 21: 1239-1248.

Gittelsohn J, Wolever TM, Harris SB, Harris-Giraldo R, Hanley AJ, Zinman B (1998) Specific patterns of food consumption and preparation are associated with diabetes and obesity in a Native Canadian community. J Nutr 128:541547.

Global Health Estimates: Deaths by Cause, Age, Sex and Country, 2000-2012 (2014) World Health Organization (WHO), Geneva. Available at:

http://www.who.int/healthinfo/global_burden_disease/estimates/en/index1.ht ml. (Accessed on June 13, 2016).

Global status report on noncommunicable diseases (2014) World Health Organization (WHO). Available at: http://www.who.int/nmh/publications/ncdstatus-report-2014/en/. (Accessed on November 25, 2015). 
Guraya SY (2015) Association of type 2 diabetes mellitus and the risk of colorectalcancer: A meta-analysis and systematic review. World J Gastroenterol 21:6026-6031.

Harris MI, Eastman RC, Cowie CC, Flegal KM, Eberhardt MS (1999) Racial and ethnic differences in glycemic control of adults with type 2 diabetes. Diabetes Care 22:403-408.

Hoffman BG, Zavaglia B, Witzsche J, Ruiz de Algara T, Beach M et al (2008) Identification of transcripts with enriched expression in the developing and adult pancreas. Genome Biol. 9:R99.

Hofmann K (2000) A superfamily of membrane-bound O-acyltransferases with implications for Wnt signaling Trends Biochem. Sci 25,pp.111-112.

Iraqi AF, Athamni H, Dorman A, Salymah Y, Tomlinson I, Nashif A, Shusterman A, Weiss E, Houri-Haddad Y, Mott R and Soller M (2014) Heritability and coefficient of genetic variation analyses of phenotypic traits provided strong basis for highresolution QTL mapping in the Collaborative Cross mouse reference population. Mammalian Genome. 25(3): 109-119.

Iraqi F, Churchill G and Mott R (2008) The Collaborative Cross, developing a resource for mammalian systems genetics: a status report of the Wellcome Trust cohort. Mammalian Genome 19: 379-381.

Iraqi FA, Mahajne M, Salaymah A, Sandovsky H, Tayem H et al (2012) The Genome Architecture of the Collaborative Cross Mouse Genetic Reference Population. Collaborative Cross Consortium. Genetics 190: 389-402.

Kirchner H, Heppner KM, Holland J, Kabra D, Tschöp MH et al (2013) Ablation of ghrelin 0-acyltransferase does not improve glucose intolerance or body adiposity in mice on a leptin-deficient ob/ob background. PLoS One 8:e61822. 
Leiter EH (2009) Selecting the "Right" mouse model for metabolic syndrome and type 2 diabetes. T2Diabetes, Methods in Molecular Biology 560: 1-17.

Levy R, Mott R, Iraqi FA, Gabetp Y (2015) Collaborative cross Mice in a Genetic Association Study Reveal New Candidate Genes for Bone Microarchitecture. BMC Genomics 16:1013: 1-14.

Lore' NI, Iraqi FA and Bragonzi A (2015) Host genotype an important determinant factor of Pseudomonas aeruginosa susceptibility in the Collaborative Cross mice. BMC Genetics 16: (106).

Mathers CD, Loncar D (2006) Projections of global mortality and burden of disease from 2002 to 2030. PLoS Med 3:e442.

Mathen PG, Thabah MM, Zachariah B, Das AK (2015) Decreased Bone Mineral Density at the Femoral Neck and Lumbar Spine in South Indian Patients with Type 2 Diabetes. J ClinDiagn Res 9:0C08-12.

MazónPeláez I, Vogler S, Strauss U, Wernhoff P, Pahnke J et al (2005) Identification of quantitative trait loci controlling cortical motor evoked potentials in experimental autoimmune encephalomyelitis: correlation with incidence, onset and severity of disease. Hum Mol Genet 14:1977-1989.

Mohlke KL, Boehnke M (2015) Recent advances in understanding the genetic architecture of type 2 diabetes. Hum Mol Genet 24:R85-92.

Montgomery MK, Hallahan NL, Brown SH et al (2013) Mouse strain-dependent variation in obesity and glucose homeostasis in response to high-fat-feeding. Diabetologia 56:1129-1139.

Otto JM, Cs-Szabó G, Gallagher J, Velins S, Mikecz K et al (1999) Identification of multiple loci linked to inflammation and autoantibody production by a genome scan of a murine model of rheumatoid arthritis. Arthritis Rheum 42:2524-2531.

Paterson AH (1995) Molecular dissection of quantitative traits: progress and prospects. Genome Research 5: 321-333. 
Philip VM, Sokoloff G, Ackert-Bicknell CL, Striz M, Branstetter L et al (2011) Genetic analysis in the Collaborative Cross breeding population. Genome Research_21:1223-1238.

Qi L, Hu FB, Hu G (2008) Genes, environment, and interactions in prevention of type2 diabetes: a focus on physical activity and lifestyle changes. CurrMol Med 8:519-532.

Qi L, Liang J (2010) Interactions between genetic factors that predict diabetes and dietary factors that ultimately impact on risk of diabetes. CurrOpinLipidol 21:31-37.

Robling AG, Warden SJ, Shultz KL, Beamer WG, Turner CH (2007) Genetic effects on bone mechanotransduction in congenic mice harboring bone size and strength quantitative trait loci. J Bone Miner Res. 22:984-991.

Rocha JL, Eisen EJ, Van Vleck LD, Pomp D (2004) A large-sample QTL study in mice: I.

Growth. Mamm Genome. 15:83-99.

Rogers LK, Bates CM, Welty SE, Smith CV (2006) Diquat induces renal proximal tubule injury in glutathione reductase-deficient mice. Toxicol Appl Pharmacol 217:289-298.

Saydah S, Cowie C, Eberhardt MS, De Rekeneire N, Narayan KM (2007) Race and ethnic differences in glycemic control among adults with diagnosed diabetes in the United States. Ethn Dis 17:529-535.

Shusterman A, Salymah Y, Nashef A, Soller M, Wilensky A, Mott R, Weiss El, Houri-Haddad $Y$ and. Iraqi FA (2013) Genotype is an important determinant factor of host susceptibility to periodontitis in the Collaborative Cross and inbred mouse populations. BMC Genetics 14: 68-79.

Soller M and Fuad Iraqi FA (2014) The Collaborative Cross - a next generation mouse genetic resource population for high resolution genomic analysis of complex traits. Livestock Sciences Journal 166: 19-25. 
Stevens JW, Khunti K, Harvey R, Johnson M, Preston L et al (2015) Preventing the progression to type 2 diabetes mellitus in adults at high risk: a systematic review and network meta-analysis of lifestyle, pharmacological and surgical interventions. Diabetes Res ClinPract 107:320-331.

Threadgill DW, Churchill GA (2012) Ten years of the Collaborative Cross. Genetics 190:291-294.

Touvier T, Conte-Auriol F, Briand O, Cudejko C, Paumelle R et al (2009) LEPROT and LEPROTL1 cooperatively decrease hepatic growth hormone action in mice. J Clin Invest 119:3830-3838.

Tracy A. Brennan, Kevin P. Egan, Carter M et al (2014) Mouse models of telomere dysfunction phenocopy skeletal changes found in human age-related osteoporosis. Disease Models and Mechanisms 7: 583-592.

van Dam RM, Rimm EB, Willett WC, Stampfer MJ, Hu FB (2002) Dietary patterns and risk for type 2 diabetes mellitus in U.S. men. Ann Intern Med 136:201-209.

vanWezel T, Ruivenkamp CA, Stassen AP, Moen CJ, Demant P (1999) Four new colon cancer susceptibility loci, Scc6 to Scc9 in the mouse. Cancer Res 59:4216-4218.

Vered K, Durrant C, Mott R and Iraqi FA (2014) Susceptibility to Klebsiella pneumonaie Infection in Collaborative Cross Mice is a Complex Trait Controlled by At Least Three Loci Acting at Different Time Points. BMC Genomics 15:865.

Welsh CE, Miller RD, Manly KF, Wang J, McMillan L et al (2012) Status and access to the Collaborative Cross population. Mammalian Genome 23:706-712.

Yalcin B, Flint J, and Mott R (2005) Using Progenitor Strain Information to Identify Quantitative Trait Nucleotides in Outbred Mice. Genetics 171: 673-681.

Yang H, Ding Y, Hutchins LN, Szatkiewicz J, Bell TA et al (2009), A customized and versatile high-density genotyping array for the mouse. Nat Methods 6:663666. 
Yang J, Brown MS, Liang G, Grishin NV, Goldstein JL (2008) Identification of the acyltransferase that octanoylates ghrelin, an appetite-stimulating peptide hormone. Cell 132:387-396.

Yan J, Meng X, Wancket LM, Lintner K, Nelin LD et al (2012) Glutathione reductase facilitates host defense by sustaining phagocytic oxidative burst and promoting the development of neutrophil extracellular traps. J Immunol 188:2316-2327. 


\section{Figures Legend:}

Fig. 1: Total area under curve (AUC0-180) of glucose clearance ( $\mathrm{min}^{\star} \mathrm{mg} / \mathrm{dL}$ ) at initial (time 0 ) and 180 minutes of intraperitoneal glucose tolerance test (IPGTT) of different CC lines in response to HF (42\% Fat) dietary challenge. Bar graph (A) shows IPGTT total AUC of 58 CC lines of overall CC lines population. Bar graph (B) shows IPGTT total AUC of females from 44 CC lines. Bar graph (C) shows total AUC of males from $56 \mathrm{CC}$ lines. $X$-axis line number, representsthe different $\mathrm{CC}$ lines. Y-axis represents the total area under curve of glucose clearance $\left(\mathrm{min}^{\star} \mathrm{mg} / \mathrm{dL}\right)$ at initial (time 0 ) and 180 minutes of intraperitoneal glucose tolerance test (IPGTT). Significant variation was found between the different $\mathrm{CC}$ lines at $P<0.001$.

Fig.2: Difference between sexes of AUC0-180 ( $\left.\mathrm{mg} / \mathrm{dL}^{*} \mathrm{~min}\right)$ means within each CC line (Total $42 \mathrm{CC}$ lines) in response to HF (42\% Fat) ditary challenge. The difference calcualted between means of AUCO-180 of males and females from the same $C C$ line $(\triangle$ AUC0-180= Males - Females). $X$-axis represents the different $\mathrm{CC}$ lines. $\mathrm{Y}$-axis represents the total area under curve of glucose clearance $\left(\mathrm{min}^{*} \mathrm{mg} / \mathrm{dL}\right)$ at initial (time 0$)$ and 180 minutes of intraperitoneal glucose tolerance test (IPGTT).

Fig. 3: Genome scan of Quantitative Trait Loci (QTL) associated with total area under curve of glucose clearance (AUCO-180 (mg/dL*min) trait in female mice, in a population of 44 Collaborative Cross (CC) lines after 12 weeks on high-fat (42\% Fat) dietary challenge. X-axis represents the 19 mouse Chromosomes and the position of mapped QTL on Chromosome 8 (Chr8). Y-axis represents the $\log P$ of the test of association between locus and AUC0-180 trait. QTL with logP exceeded 5.65 threshold, based on permutaion genome wide test, at significant level of $\mathrm{P}<0.05$ was identified.

Fig. 4: Estimated effect size on total area under curve of glucose clearance (AUC0-180) $\left(\mathrm{mg} / \mathrm{dL}^{*} \mathrm{~min}\right)$ ) for the $8 \mathrm{CC}$ founder strains for Chromosome $8 \mathrm{QTL}$. $\mathrm{X}$-axis represents eight founder strains of the $\mathrm{CC}$ mice. Y-axis represents haplotype effect size of the CC founder at the AUC0-180 QTL.

Fig. 5: Merge analysis of sequence variants around the AUC0-180 QTL on Chromosome 8 in the Collaborative Cross (CC) lines. X-axis represents the genomic location. Y-axis represents the logP of the linkage analysis between AUC0-180 and the locus. The orange dots resemble the results of merge analysis. The blue continues line resemble the haplotype QTL mapping scan on Chromosome 8. 
Table 1: Position of the QTL underlying the total area under curve of glucose clearance trait (AUC0-180 QTL) associated with impaired glucose tolerance in response to 12 weeks HF (42\% Fat) dietary challenge in Collaborative Cross (CC) lines. QTL; Quantitative Trait Loci, Chr; Chromosome, -logP- negative logarithm of significance value, $\mathrm{H}^{2}$, estimated broad-sense heritability, CVg; coefficient of genetic variation. 
Figure 1. Phenotypic profile of AUC0-180 of total CC population (A), male, only (B) and female, only (C).

A.

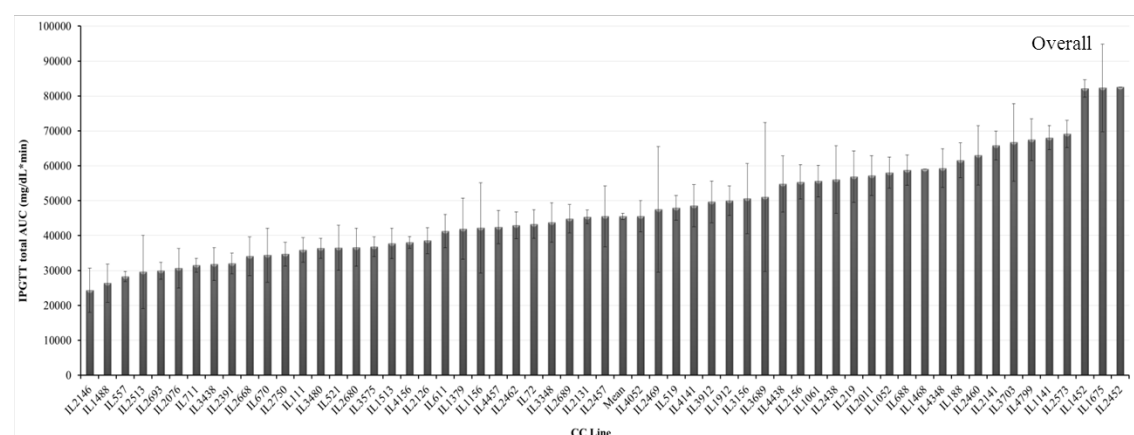

B.

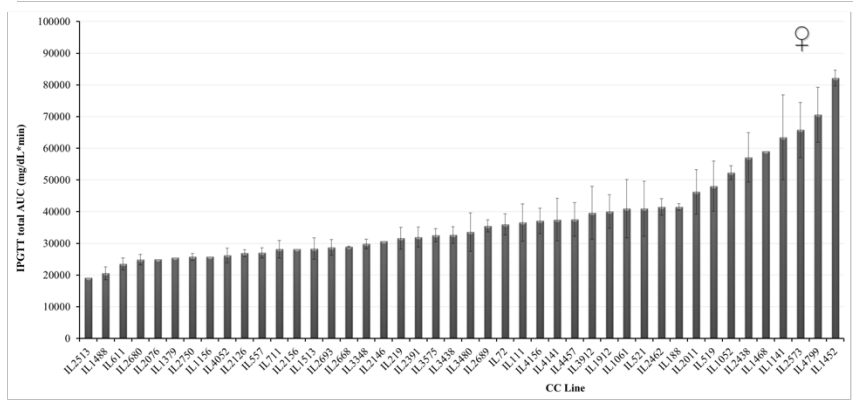

C.

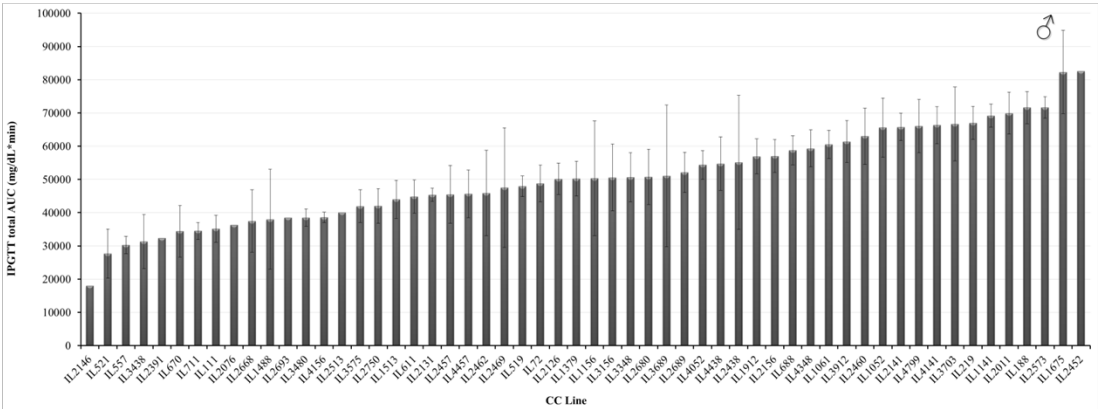


Figure 2: Differences of AUC0-180 between males and females CC mice

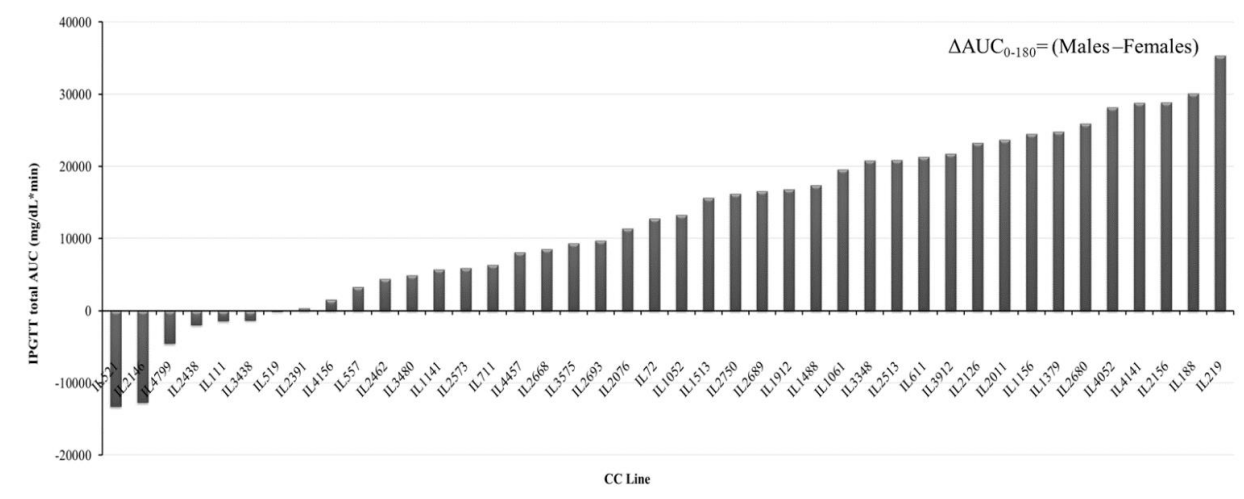


Figure 3. Mapped QTL with AUC0-180 trait

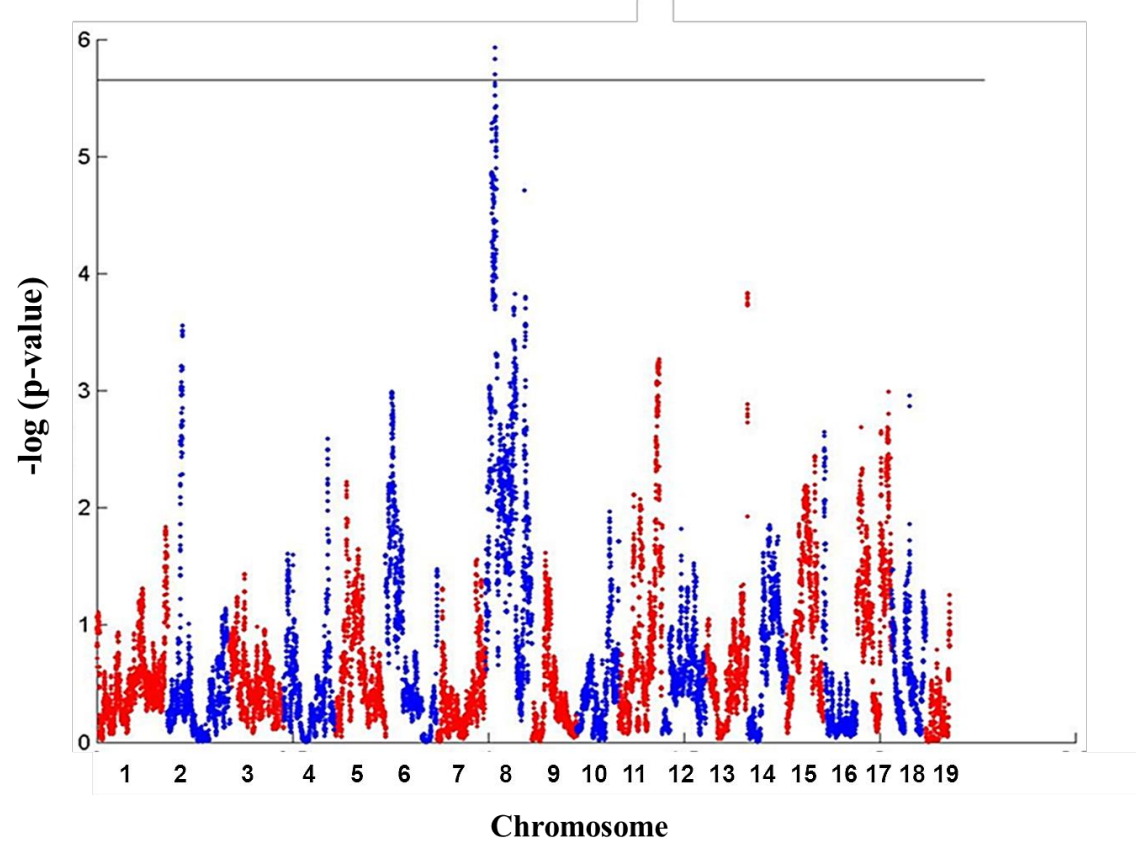


Figure 4. Founder Effect

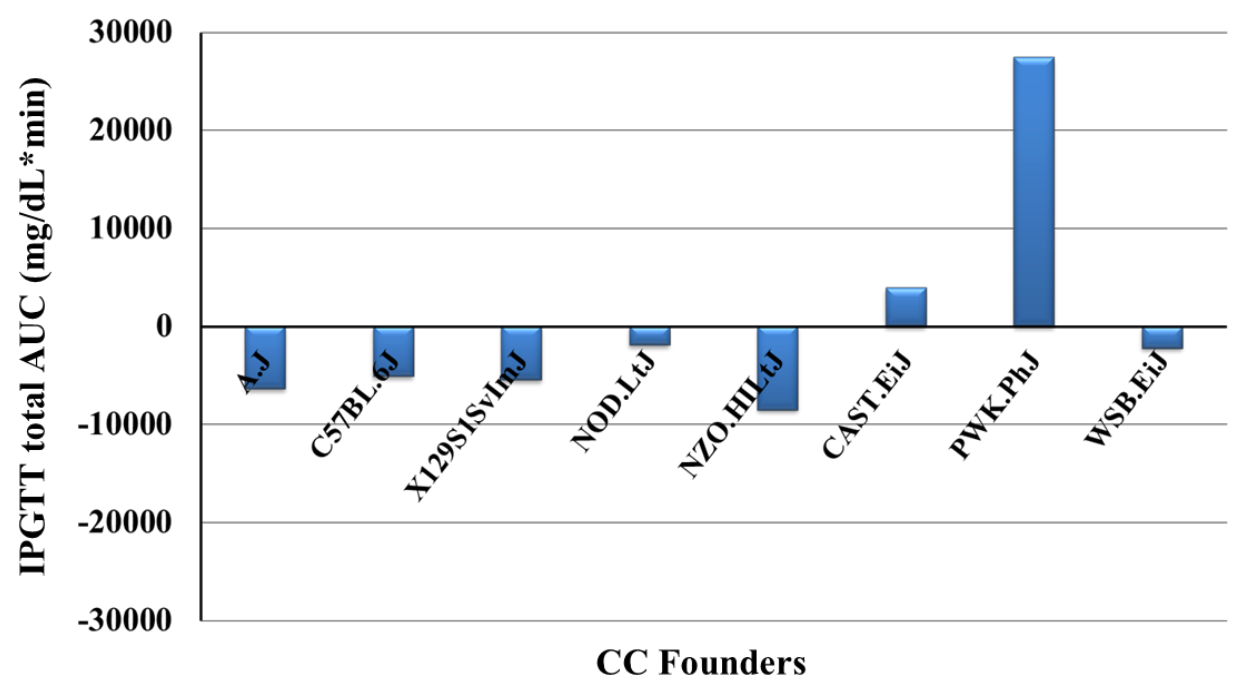


Fig. 5: Merge analysis

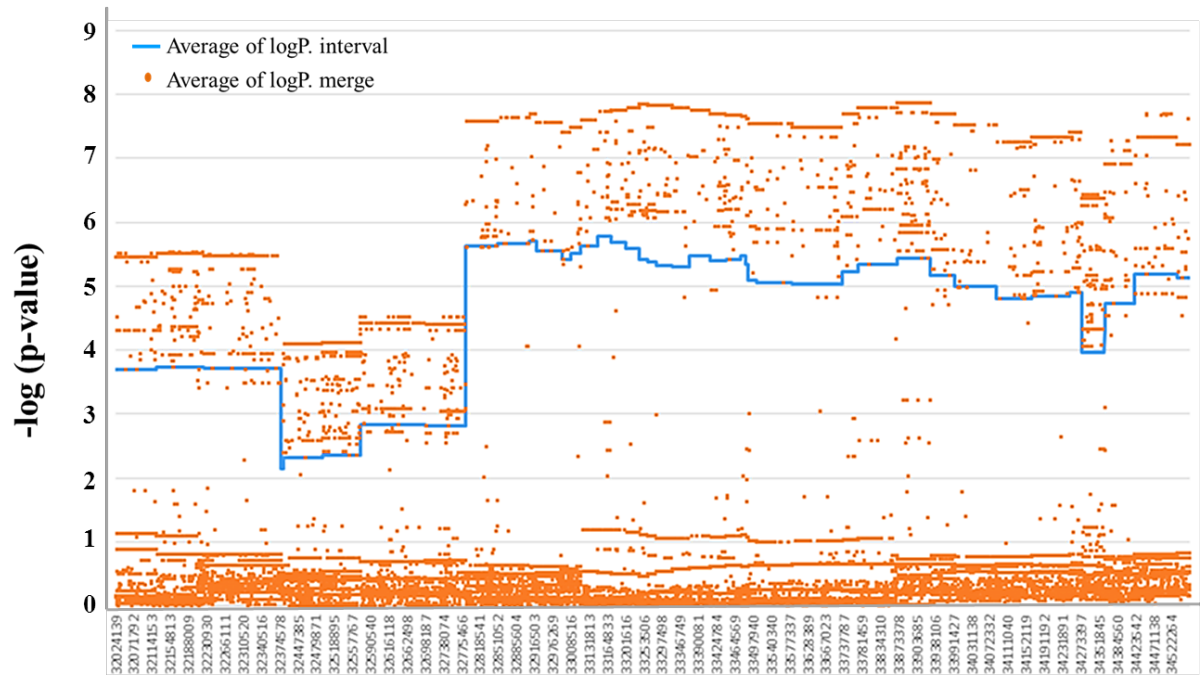

Genomic Location 